\title{
In situ Studies of Catalytic Growth of SiC Nanowire
}

Yihong Kang, ${ }^{1}$ Penghan Lu, ${ }^{2}$ Xiaofeng Zhang, ${ }^{3}$ Zhenyu Liu, ${ }^{4}$ JL Sturgeon, ${ }^{5}$ John Mastovich, ${ }^{5}$ Zhiwei Shan, ${ }^{2}$ Judith C. Yang, ${ }^{6}$

1. Mechanical Engineering and Materials Science, University of Pittsburgh, Pittsburgh, PA 15261, USA

2. Center for Advancing Materials Performance from the Nanoscale, Xi'an Jiaotong University, Xi'an, Shanxi, China

3. Nanotechnology System Division, Hitach High Technologies America, Pleasanton, CA 94588 USA

4. Kennametal Inc., Latrobe, PA 15650, USA

5. RL Lee Group Inc., Monroeville, PA 15146, USA

${ }^{6 .}$ Chemical and Petroleum Engineering, University of Pittsburgh, Pittsburgh, PA 15216, USA

Nanoscale materials are currently being exploited as active components in a wide range of technological applications in different fields, such as composite materials, chemical sensing, biomedicine, optoelectronics and nanoelectronics. Many recent efforts have focused on the creation of various nanostructures including spheres, tubes, cables, sheets and, especially wires, since those nanoscale building blocks are anticipated to transform the semiconductor industry[1]. However, the realization of practical nanodevices requires that these nanostructures be integrated efficiently and economically into various intricate architectures. Synthetic control of onedimensional (1D) nanostructures into complex heterostructures would impact the design of future electronic devices by simplifying much of the processing associated with nanodevice fabrications.

Among the various semiconductors, silicon carbide ( $\mathrm{SiC}$ ) is an important group IV-IV compound that possesses unique physical and electronic properties. SiC-based electronics will be better than silicon-based devices in certain applications, because they can operate at higher temperature, higher power, and higher frequency as well as withstand harsh environments but with reduced cooling requirements. A lot of studies showed the effort people put into the controlled synthesis of $1 \mathrm{D} \mathrm{SiC} \mathrm{nanowire[2].} \mathrm{Fe} \mathrm{catalyst} \mathrm{was} \mathrm{used} \mathrm{widely} \mathrm{in} \mathrm{the} \mathrm{synthesis} \mathrm{of} \mathrm{SiC} \mathrm{nanowire,} \mathrm{but} \mathrm{the}$ role of the catalyst during the reaction is still unknown, therefore, there is no precise control of the 1D structure and other more useful structures, such as cone-structure, Y junction and even $\mathrm{T}$ junctions. We investigated catalytic role of Fe during the formation of $\mathrm{SiC}$ nano-cone for gaining insights into the control of catalytic synthesis of $\mathrm{SiC}$ nano-structure using environmental transmission electron microscopy (ETEM) and analytic scanning transmission electron microscopy (STEM). Previous studies show the synthesis of SiC nanowire using Fe encapsulated in carbon shell (Fe@C) and $\mathrm{SiO}$ vapor around $1300^{\circ} \mathrm{C}[3,4]$, but both nanocone with Fe catalyst on the top and nanocone without Fe catalyst on the top, as well as some complex structures were observed as shown in Figure 1. The vapor-liquid-solid model, first proposed by Wagner and Ellis[5], is the presently accepted descriptive paradigm of the nanowire formation. We speculate that either the agglomeration of Fe particles creates those complex 1D and branched or junction structures[3, 4], or the solidification and segregation of $\mathrm{SiC}$ from large Fe-C-Si ternary pieces as 
the non-catalytic process[6]. Hence, we studied the formation of $\mathrm{SiC}$ nano-structure in the Hitachi H-9500 that permits heating as high as $1300^{\circ} \mathrm{C}, 2 \mathrm{~Pa}$ Ar pressure. This instrument is a $300 \mathrm{KV} \mathrm{Lab} 6$ atomic resolution transmission electron microscope (TEM) equipped with high temperature throughput, gas injection system and high stability 5-axis side-entry hyper stage. Critical examination of the formation of SiC complex 1D structure will provide important insights into the nucleation and evolution mechanism of those unusual nanostructures needed to build nanodevices based on 1D structures and their derivates, such as $\mathrm{Y}$ - and T- junctions.

This research was supported in part by a grant from the NSF (DMR-0804892).

Reference

[1] L. Manna, D.J. Milliron, A. Meisel, E.C. Scher, A.P. Alivisatos, Controlled growth of tetrapodbranched inorganic nanocrystals, Nat Mater, 2 (2003) 382-385.

[2] W. Zhou, Y. Zhang, X. Niu, G. Min, One-Dimensional SiC Nanostructures: Synthesis and Properties, in: Z.M. Wang (Ed.) One-Dimensional Nanostructures, Springer, 2008, pp. 330.

[3] Z. Liu, C. Lijie, V. Srot, N.Y. Jin-Phillipp, P.A. van Aken, M. Rühle, J.C. Yang, Crystalline silicon carbide nanocones and heterostructures induced by released iron nanoparticles, Appl. Phys. Lett., 93 (2008) 233113.

[4] Z. Liu, V. Srot, J.C. Yang, Self-assembled crystalline silicon carbide Y junctions by coalescence of nucleated iron catalysts, Appl. Phys. Lett., 96 (2010) 253111.

[5] R.S. Wagner, W.C. Ellis, VAPOR-LIQUID-SOLID MECHANISM OF SINGLE CRYSTAL GROWTH, Applied Physics Letters, 4 (1964) 89-90.

[6] J. Sulistyo, T. Hata, S.N. Marsoem, Formation of $\mathrm{SiC}$ Rods in Composites of $\mathrm{SiC} / \mathrm{SiO} 2 / \mathrm{C}$ from Carbonized Wood Infiltrated with Ethylsilicate-40, Wood Research Journal, 2 (2011).
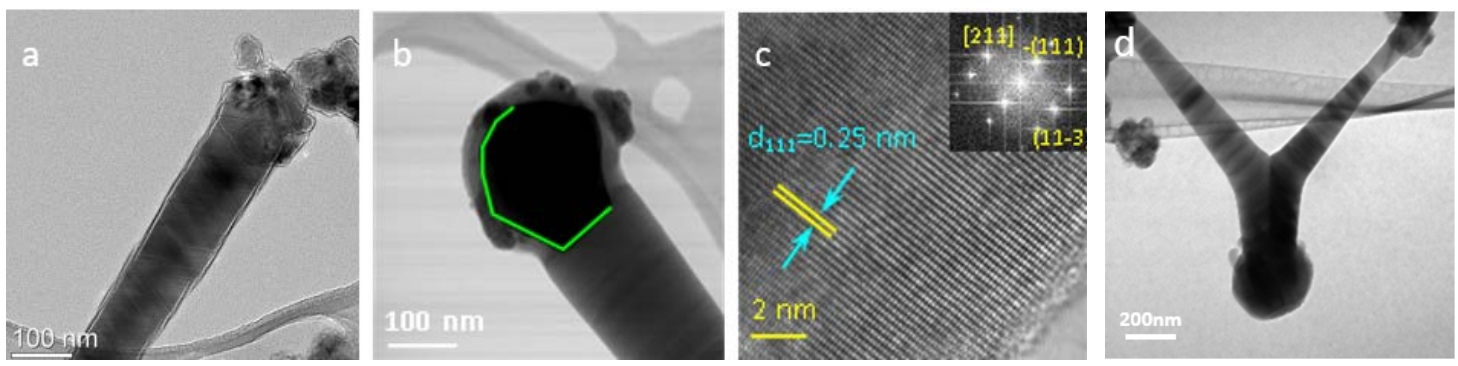

Figure 1. Images of $\mathrm{SiC}$ nanocwire. (a) $\mathrm{SiC}$ nanowire without a Fe catalyst on the top of it, (b) $\mathrm{SiC}$ nanowire with a Fe catalyst on the top of it, (c) High-resolution image of SiC nanowire showing the $<111>$ growth direct, (d) Y junction with two inclined branches to form a stem with a planar grain boundary along the stem center and corresponding change of the growth direction.
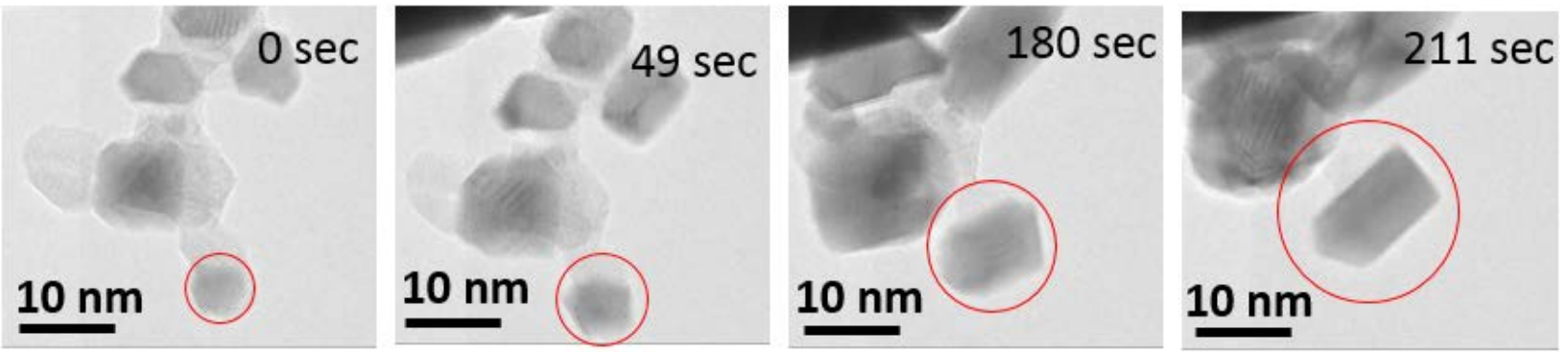

Figure 2. The growth of $\mathrm{SiC}$ piece under $1400^{\circ} \mathrm{C}, 2 \mathrm{~Pa}$ Ar pressure. 